Analyse factorielle multiple avec $R$ 


\section{KVSDJHIQWQWRQDCIOHKEOQN}


Jérôme Pagès

Analyse factorielle multiple avec $\mathrm{R}$ 
ISBN : 978-2-7598-0963-9

(C) 2013, EDP Sciences, 17, avenue du Hoggar, BP 112, Parc d'activités de Courtabœuf, 91944 Les Ulis Cedex A

Imprimé en France

Tous droits de traduction, d'adaptation et de reproduction par tous procédés réservés pour tous pays. Toute reproduction ou représentation intégrale ou partielle, par quelque procédé que ce soit, des pages publiées dans le présent ouvrage, faite sans l'autorisation de l'éditeur est illicite et constitue une contrefaçon. Seules sont autorisées, d'une part, les reproductions strictement réservées à l'usage privé du copiste et non destinées à une utilisation collective, et d'autre part, les courtes citations justifiées par le caractère scientifique ou d'information de l'œuvre dans laquelle elles sont incorporées (art. L. 122-4, L. 122-5 et L. 335-2 du Code de la propriété intellectuelle). Des photocopies payantes peuvent être réalisées avec l'accord de l'éditeur. S'adresser au : Centre français d'exploitation du droit de copie, 3, rue Hautefeuille, 75006 Paris. Tél. : 0143269535. 


\author{
Collection Pratique $\mathbf{R}$ \\ dirigée par Pierre-André Cornillon \\ et Eric Matzner-Løber \\ Département MASS \\ Université Rennes-2-Haute-Bretagne \\ France
}

\title{
Comité éditorial
}

\section{Eva Cantoni}

Institut de recherche en statistique

\& Département d'économétrie

Université de Genève

Suisse

\section{François Husson}

Département Sciences de l'ingénieur

Agrocampus Ouest

France

\section{Pierre Lafaye de Micheaux}

Département de Mathématiques et Statistique

Université de Montréal

Canada

\section{Sébastien Marque}

Directeur Département Biométrie

Danone Research, Palaiseau

France

Déjà paru dans la même collection :

Psychologie statistique avec $R$

Yvonnick Noël, 2013

ISBN : 978-2-8178-0425-5 - Springer

Séries temporelles avec $R$

Yves Aragon, 2011

ISBN : 978-2-8178-0208-4 - Springer

Régression avec $R$

Pierre-André Cornillon, Eric Matzner-Løber, 2011

ISBN : 978-2-8178-0184-1 - Springer

Méthodes de Monte-Carlo avec $R$

Christian P. Robert, George Casella, 2011

ISBN : 978-2-8178-0181-0 - Springer 


\section{KVSDJHIQWQWRQDCIOHKEOQN}

